\title{
Recommending coronary artery surgery: refining judgement through application of new knowledge
}

\author{
J. F. GoodwIN \\ M.D., F.R.C.P., F.A.C.C. \\ Royal Postgraduate Medical School, Hammersmith Hospital, London W12 OHS
}

\begin{abstract}
Summary
An analysis is made to help decide on the best form of treatment for atherosclerotic coronary disease. Available data on surgical treatment are reviewed with emphasis on prognosis and management of the patient with stable angina.
\end{abstract}

\section{Introduction}

Between the challenges of prevention of atherosclerotic coronary artery disease and its complications on the one hand, and the performance of complex arterial reconstruction by surgical means on the other, lies a crucial area for the patient and his doctor. Prevention still presents a formidable long term problem, while the remote effects of coronary artery bypass grafting are still unknown. The patient who suffers from the disease needs immediate expert advice about the best form of treatment available to him at the present time, and for this the physician must be prepared to make a decision based on evidence that is often incomplete.

The following analysis is an attempt to arrive at such a decision by reviewing the available data on surgical treatment for coronary artery disease. The main emphasis will be on the prognosis and management of the patient with stable angina (with or without previous cardiac infarction) in order to evaluate the place of surgical treatment at the present time. No attempt will be made to be fully comprehensive regarding the therapy of ischaemic heart disease.

The main problem and important concepts of this 'coronary dilemma' may be stated as follows:

(1) Coronary artery bypass grafting offers a high chance (around $80 \%$ ) of full or good relief of symptoms. The effect of the operation on prognosis is still uncertain. The twin aspects, symptomatic relief and prognosis, are separate but interdependent and both must be considered when evaluating the best treatment for the individual.

(2) Treatment should be planned as a programme and not as a single event. Over a prolonged course of time, surgical treatment may be added to medical treatment.

(3) Attention to factors which are believed to be important must never be neglected in favour of exciting feats of surgery. Of particular importance are the avoidance of cigarette smoking and the control of hypertension and lipid disorders.

In order to examine the problem, several aspects should be considered.

\section{The prognosis of angina}

The annual mortality of angina varies from $2 \%$ in uncomplicated cases to $10 \%$ when complications such as hypertension or persistent electrocardiographic changes are present. The 10-year survival can be as high as $60 \%$, or as low as $25 \%$ when persistent ECG changes are present (Block et al., 1952).

While predictions based on hypertension, previous infarction and persistent electrocardiographi changes are valuable, more accurate information cap be obtained by relating prognosis to the degree of coronary artery disease and to the severity of left ventricular dysfunction assessed by angiography. Numerous studies from the U.S.A. have produced fairly general agreement that for significant disease of the three main coronary arteries the annual mortality is around $20 \%$ and the 10 -year mortality over 50\% (Humphries et al., 1974; Burggraf and Parker, 1975).

With disease of two vessels, 4-year mortality is appreciably better at $80 \%$ but 10 -year mortality parallels that of 3-vessel disease. Single-vessel disease carries a prognosis of $80 \%$ survival at 10 years and $90 \%$ survival at 1 year, but this mortality figure is apparently mainly due to disease of the left anterior descending artery, as isolated disease of the right coronary artery carries a near $100 \%$ survival at 10 years (Burggraf and Parker, 1975).

The prognosis of stenosis of the left main coronary artery is now considered to be poor and the risks to life considerable. The risks may be as great or perhaps greater than that of severe 3-vessel disease, with a greater additional element of uncertainty, so that the patient is at serious risk of sudden death or massive left ventricular infarction at any time (Burggraf and Parker, 1975).

Survival is also related to the presence of heart 
failure, which is associated with less than 8 years' survival in $80 \%$ of patients (Burggraf and Parker, 1975).

Thus, it is clear that angina with heart failure and 2- or 3-vessel disease carries a very poor prognosis and that the chance of survival for more than 5 years are poor. Conversely, single-vessel disease without heart failure appears benign, but it is important to bear in mind that the invariable tendency for coronary disease is to progress and that the prognosis may deteriorate as occlusive disease spreads in the coronary system and the function of the left ventricle deteriorates as a result. The development of collateral vessels will not necessarily redress the balance.

It should be remembered that all assessments of prognosis based on coronary arteriographic findings assume the prognosis from the time of diagnosis. There is no way of knowing for how many years before coronary arteriography the patient may have had severe multiple disease and so prognosis may sometimes be unduly gloomy. The tendency for coronary disease to progress and the evidence obtained from serial angiographic studies would suggest that the figures for survival are probably approximately correct.

\section{The effect of angina on left ventricular function}

It has long been known that anginal pain is accompanied by evidence of left ventricular dysfunction. Elevation of the left atrial and left ventricular end-diastolic pressures has been shown, and regional dyskinesia has been demonstrated (Sharma et al., 1975). It is uncertain whether angina precedes left ventricular failure or vice versa. The development of left ventricular failure could well precipitate angina by increasing wall-tension and oxygen consumption as a result of ventricular dyskinesia and dilatation. Recent work in the laboratory at the Royal Postgraduate Medical School suggests that left ventricular dysfunction becomes severe when exercise precipitates angina, but is only slightly impaired when exercise does not lead to anginal pain. Sharma et al. (1975) have shown by angiography that striking increases in left ventricular end-diastolic volume occur in patients with coronary artery disease who are exercised to the point of angina. By contrast, other patients with coronary artery disease in whom exercise did not produce angina, showed only slight abnormalities of left ventricular function. Dyskinesia became prominent in the anginal group but not in those without angina. These findings would suggest that angina, in addition to being a painful and disabling symptom, is associated with damage to the left ventricle, which could be permanent or progressive, in patients in whom it is uncontrolled. If so, this would link closely the symptoms and the prognosis of angina in patients with ischaemic heart disease and would indicate the importance of preventing angina whenever possible, though not necessarily by surgical means.

The effect of coronary artery bypass grafting on symptoms and prognosis: comparison with medical treatment

It is not the purpose of this communication to deal with the symptomatic results of coronary artery bypass grafting, for it can be assumed that around $80 \%$ of patients will obtain good relief of symptoms (Cannon et al., 1974). The effect on prognosis is far less certain and depends on many factors. In skilled hands the mortality of the operation should te around $2 \%$ for uncomplicated patients or even lower. Graft patency may be expected to persist for up to 5 years in $80 \%$ of patients, provided that the initial flow is adequate. The course of 960 patients followed up to 4 years after operation has been reported by Sheldon et al. (1973), the survival rate at 4 years was over $90 \%$. These workers compared this group with a group of 469 patients with 1-, 2-, and 3-vessel disease, respectively, treated medically and found their survival to be just over $70 \%$. Although this study demonstrated an advantage of surgical over medical treatment, the series were not contemporary, patients were not randomized and the medically treated group were retrospective. Also, differences between survival depending on the extent of the disease could not be assessed. While of great value it is doubtful whether these data can be used effectively to compare medical and surgical treatment. Another valuable study, which also suffered from the same disadvantages, was that of Cannon et al. (1974), in which the surgical group was compared with two unrelated series treated medically. The surgical group's survival rate was less than each medical group for the first 16 months after the operation but at 36 months had exceeded the medical by a few per cent.

Both these studies demonstrate the difficulties of assessing the effect of the operation on prognosis and emphasize the early higher mortality in the surgical group, presumably due to the operative risks, even though these are small. Thus, the benefits of the operation on prognosis are essentially remote in most cases and so the potential benefits of surgery on prognosis should be regarded as mainly against sudden death, recurrent infarction and progressive irreversible ventricular damage.

The data gained from coronary arteriography would suggest that the operation will not improve prognosis in single-vessel disease (especially in isolated right coronary disease) and may even worsen it. However, the prognosis of 3-vessel disease appears to be such that operation is likely to improve 
prognosis. Significant stenosis of the left main coronary artery should be regarded as a threat to life and therefore probably an indication for urgent operation. The author agrees with Kirklin (1974), that 'present information does not support advising patients with single-vessel disease to have operation solely for prolonging life, but does make such advice rational in patients with triple-vessel disease'. In weighing the pros and cons of operation it must be remembered that those who have the least operative risk may have the least need of the operation and probably the least to gain. Those who have the greatest need (severe 3-vessel disease and impaired left ventricular function or heart failure) have the highest risks of surgery but the most to gain. It seems very likely that operation, if the patient survives, will be expected to prolong life in those with heart failure or severely compromised left ventricular function. At what exact point compromised left ventricular function becomes too severe to allow a favourable outcome is uncertain, but it has been suggested that if angina persists operation may be undertaken, although with a considerably increased risk, but should not be advised when there is heart failure due to ischaemic disease in the absence of angina. The presence of angina implies that there is viable but ischaemic muscle which can possibly be rescued by revascularization. Experience at the Royal Postgraduate Medical School in our department indicates that successful coronary artery bypass grafting can produce impressive improvement in ventricular function, especially when tested on effort (Sharma et al., 1975). Dyskinesia improves, ventricular volumes diminish and ejection fractions increase.

The relative merits of medical and surgical treatment in improving prognosis can really only be assessed by prospective randomized trials on a large scale such as the American Veterans Administration trial discussed in this symposium (Hultgren, 1976). A similar multicentre European trial is in progress to which twelve centres in Europe contribute patients. Patients admitted into the trial must be male, less than 65 years of age, have suffered from cardiac infarction more than 2 months previously and must be suffering from significant stable angina. They must have important coronary artery disease involving at least two major arteries and the lesions must be amenable to successful bypass grafting. Ventricular function must be 'reasonable'. Results of this trial are still awaited. A trial on unstable angina, designed along similar lines, is also in progress in the U.S.A.

\section{Effects of long term $\beta$-adrenergic blockade on prog- nosis}

The place of $\beta$-adrenergic blocking agents in cardiovascular disease with special reference to ischaemic heart disease has been dealt with extensively in this symposium (Fitzgerald, 1976). It is worth reiterating the importance of data now emerging that suggests a beneficial effect on prognosis of ischaemic heart disease from $\beta$-adrenergic blockade. Wilhelmsson et al (1974) showed a significant reduction in the instance of sudden death (but not in nonfatal infarction) in a 2-year double-blind study of patients treated with alprenolol after an acute ischaemic episode. Fox et al. (1975) showed a reduction in the incidence of infarction in patients who were on $\beta$-adrenergic blockade at the time of admission to a coronary care unit for an acute coronary episode. There was no reduction in the incidence of death. These two studies were of course measuring different aspects of prognosis, but the results, and those quoted by Fitzgerald (1976), give an indication of hope for medical prophylaxis which must be kept in mind when evaluating the role of surgery in prognosis.

\section{Adverse effects of coronary artery bypass grafting}

There are three main problems: occlusion of the graft; progression of the underlying coronary artery disease; post-operative myocardial infarction and deterioration in left ventricular function.

\section{Gratt occlusion}

Between 70 and $85 \%$ of grafts are patent 1-2 year after operation. Early patency depends on adequate flow through the graft at the time of the operation. Late occlusion is a result of structural changes in the vein, probably fibrous hyperplasia (Bourassa et al., 1972; Lespérance et al., 1973). The incidence of occlusion levels-off after the first post-operative year, suggesting that fibrous hyperplasia does not progress significantly after this time (L'Esperance et al., 1973).

Occlusion of the graft is usually accompanied by a brisk return of angina, which nullifies the symptomatic benefits of the operation. Worse still, the occlusion can impair left ventricular function, so $15-30 \%$ of patients run a risk of being made worse by the operation. It is interesting to speculate whether there may be two types of patient, as has been suggested for occlusive peripheral arterial disease: one group of younger patients who have progressive vascular disease and in whom grafts block readily and early, and another group of older patients who have a more stable disease and in whom grafts remain patent for longer. The indications for operations could be more readily assessed if future work elucidates this aspect of the problem. Although the available evidence does not warrant a conclusion that coronary artery bypass grafts themselves improve left ventricular function, functioning grafts have been shown to participate directly in the mechanical events of the cardiac cycle, which may 
explain the improvement in regional dyskinesia found after successful bypass grafting. Moran et al. (1973) concluded that successful bypass appeared to contribute effectively to the maintenance of myocardial contractility in the revascularized area.

\section{Progress of underlying coronary artery disease}

Since the natural tendency of occlusive atherosclerotic coronary artery disease is to progress, it is not surprising that this should happen after coronary artery bypass grafting. Possibly a successful graft diverting blood flow from the post-stenotic segment, may accelerate occlusion by a 'steal' mechanism (Bousvaros et al., 1972). It is not known whether new patterns of flow set up at the sight of the graft insertion might accelerate the progress of the underlying disease.

In a study of 100 patients, Malinow et al. (1973) found that proximal coronary artery stenosis progressed to complete occlusion in nearly $50 \%$ of the patients who had a graft to stenosed arteries. The incidence was considered higher than that of nongrafted arteries in the natural course of the disease. Occlusive lesions in the underlying coronary circulation occurred more often with patent than with occluded grafts. Bemis et al. (1973) found that occlusive disease of the coronary circulation advanced more rapidly in patients with lipid abnormalities and noted that treatment of the lipid disorders did not influence the atherosclerotic occlusions. Levine et al. (1975) considered that adverse effects of coronary artery bypass grafting occur early after operation and are due to loss of the proximal segments of grafted arteries, which may not impair the total blood flow in the distribution of the grafted vessel. Occlusion of distal coronary arteries was always considered to be due to advance of the underlying disease.

The evidence would seem to suggest that coronary artery bypass grafting does not improve the underlying occlusive coronary artery disease and may possibly worsen it. In most instances, however, revascularization has little influence on the underlying vascular disease.

\section{Postoperative myocardial infarction and the effect of coronary artery bypass grafting on myocardial func- tion}

Electrocardiographic appearances indicative of myocardial infarction appear in 7-20\% of patients after coronary artery bypass grafting (Kouchoukos, Kirklin and Oberman, 1974) but enzyme changes appear in as much as $80 \%$ of patients (Oldham et al., 1973). However, these abnormalities may be inherent in cardio-pulmonary bypass operations for Kouchoukos et al. (1974) reported an incidence of electrocardiographic evidence of infarction in $13 \%$, and isoenzyme evidence of myocardial cellular damage in $83 \%$, of patients following aortic valve replacement.

The effect of coronary artery bypass grafting on myocardial function as distinct from that on the underlying coronary arteries is variable. As has been noted the occlusion of the graft is likely to be followed by deterioration in function, but impairment of segmental left ventricular contractility has been reported when the graft supplying the area remained patent (Shepherd et al., 1974). These workers concluded that coronary artery bypass grafting should not be advised merely to improve left ventricular function. While it is certain that successful bypass grafting is capable of improving left ventricular function the likelihood of its doing so in a given patient cannot be predicted. Solignac, Guerat and Bourassa (1975) found that pre-operative left ventricular dysfunction at rest was not usually improved after operation but that normal preoperative left ventricular function tended to remain normal for 3-4 years after operation. They considered that pre-operative ventricular function exerted an important influence on survival and that operations should not be delayed until left ventricular function had deteriorated substantially.

The variable effects of operation on left ventricular function at rest indicate the need for more refined studies involving the assessment of function on effort and also after measures which may improve function, such as the use of glyceryl trinitrate, both and after operation.

The present pesition of coronary artery bypass grafting for ischaemic heart disease may be summed up therefore as follows:

(1) Persistent significant angina remains the basic indication for the operation.

(2) In selected cases angina is relieved in $80 \%$ of patients: surgical expertise and technique are extremely important.

(3) Relief of angina may or may not be accompanied by improvement or deterioration of left ventricular function for reasons not fully understood.

(4) The bypass may become occluded in $20 \%$ of patients, leading to a return of angina and to deterioration in left ventricular function.

(5) The underlying coronary disease is not improved by coronary artery bypass grafting.

(6) The effect on prognosis awaits the result of trials now in progress, but it is reasonable to suppose that operation may improve prognosis in patients with 3-vessel disease, and probably with significant 2-vessel disease, if the usual indications for operation are observed. Prognosis will not be improved in single-vessel disease.

(7) The possibility that long term treatment with 
TABLE 1. The coronary dilemma 1975. Attitudes to coronary arteriography and coronary artery bypass grafting (CABG)

All patients with ischaemic heart disease should have coronary arteriography, and if suitable CABG

No

Only patients with severe, intractable, persistent, life-mutilating angina should be considered for CABG

All with angina should have coronary arteriography to provide maximal information about their disease (i.e. single-vessel disease)

The severity of angina is not related to the severity or prognosis of the disease: persistence of angina is a better guide. Persistence is therefore the best indication for coronary arteriography

The incidence of apparently 'normal' coronary arteries associated with angina widens the indications for coronary arteriography

$\beta$-adrenergic blocking drugs may improve prognosis significantly must be constantly borne in mind.

(8) Whether or not surgery is advised, constant attention must be given to risk factors, especially cigarette smoking and hypertension.

(9) The figures for surgical results quoted tend to reflect the best available surgical expertise. In less experienced hands, the disadvantages and problems of the operation are expected to be greater. Surgical expertise is a most important ingredient for success.

(10) Operation should be advised in patients with continuing angina and severe 2- or 3-vessel disease. Poor left ventricular function greatly increases the risk, but even so ventricular function may be im- proved if the operation is successful and the patient survives.

(11) The place of coronary artery bypass grafting in 'unstable angina' and in impending or evolving myocardial infarction remains uncertain.

Bearing these conclusions in mind, the following $\frac{C}{0}$ questions are important and should be answered (Table 1).

Having regard to the answers given in Table 1, the following 'flow chart' is recommended (Fig. 1). It ${ }^{\text {क }}$ must be emphasized that the guidelines given are $\vec{\circ}$ tentative, are based on personal views and cannot $\vec{\overrightarrow{ }}$ be regarded as final in a field which is constantly changing and in which so much remains to be learnt. Every patient must be considered on individual merits with regard to all relevant factors.

The future may well reveal that coronary artery ? bypass grafting only improves prognosis in certain well defined groups and may be definitely contraindicated in others. It is likely to retain an important place in treatment but that place may turn out to be limited. The identification of certain subjects for which operation is indicated or contra-indicated is the challenge for the cardiologist in the next decade.

\section{References}

ANDERSON, R.P. (1972) Effects of coronary artery bypas occlusion on left ventricular performance. Circulation, 40 507.

Bemis, C.E., Gorlin, R., Kemp, H.G. \& Herman, M.V. (197/3 Progression of coronary artery disease. A clinical arteriographic study. Circulation, 47, 455.

Block, W.J., Jr, CRumPaCker, E.L., DRY, T.J. \& GAGE, R.P. (1952) Prognosis of angina pectoris. Observations in 6882 cases. Journal of the American Medical Association, 150, 259.

TABLE 2. Management of the patient with coronary heart disease, 1975

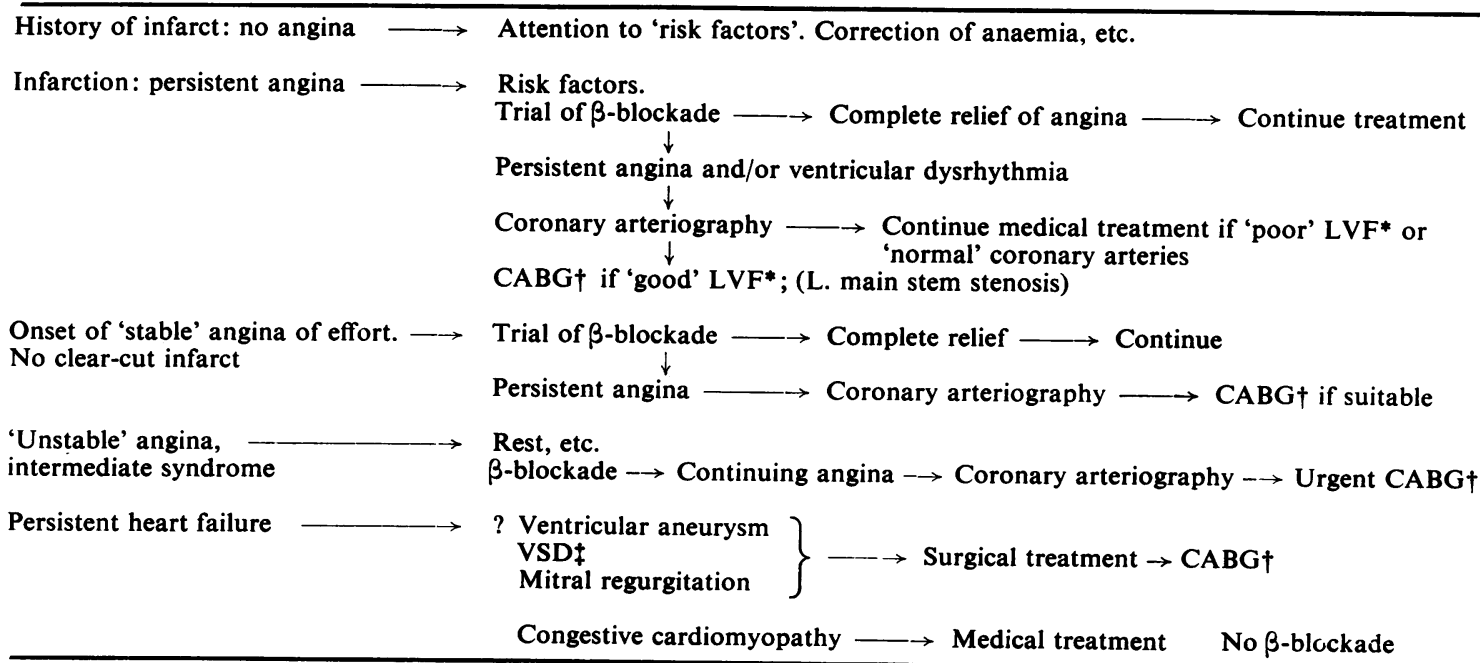

\footnotetext{
* Left ventricular function; $\uparrow$ coronary artery bypass grafting; $\ddagger$ ventricular septal defect.
} 
Bourassa, M.G., L'Esperance, J., Campeau, L. \& Sinard, P. (1972) Factors influencing patency of aorto-coronary vein graft. Circulation, 45 (Suppl. 1), 79.

Bousvaros, G., Piracha, A.R., Chaudhry, M.A., Grant, C., Older, T.M. \& PIfARRe, R. (1972) Increase in severity of proximal coronary disease after successful aortocoronary grafts; its nature and effects. Circulation, 46, 870.

Burggraf, G.W. \& Parker, J.O. (1975) Prognosis in coronary artery disease. Angiographic, hemodynamic and clinical factors. Circulation, 51, 146.

Cannon, D.S., Miller, D.C., Shumway, N.E., Fogarty, T.J., Daily, P.O., Hu, M., Brown, B., Jr \& Harrison, D.C. (1974) The long-term follow up of patients undergoing saphenous vein bypass surgery. Circulation, 49, 77.

FitzGerald, J.D. (1976) The effect of $\beta$-adrenoreceptive antagonists on the morbidity and mortality in cardiovascular disease. Postgraduate Medical Journal, 52, 770.

Fox, K.M., Chopra, M.P., Portal, R.W. \& ABer, C.P. (1975) Long term beta blockade: possible protection from myocardial infarction. British Medical Journal, 1, 117.

Hultgren, H.N., Takaro, T., Detre, K. \& Participants in the Veterans Administration CoOperative Study (1976) Medical versus surgical treatment of stable angina pectoris: progress report of a large scale study. Postgraduate Medical Journal, 52, 757.

Humphries, J.O., Kuller, L., Ross, R.S., Freisinger, G.C. \& PAge, E.E. (1974) Natural history of ischemic heart disease in relation to arteriographic findings. Circulation, 49, 489.

KIRKLIN, J.W. (1974) Surgery for aorto-coronary heart disease. In: Year Book of Cardiovascular Medicine and Surgery, p. 32. Year Book Medical Publishers, Chicago.

Kouchoukos, N.T., Kirklin, J.W. \& Oberman, A. (1974) An appraisal of coronary bypass grafting. Circulation, 50, 11.

lespérance, J., Bourassa, M.G., Saltiel, J., Campeau, L. \& Grandin, C.M. (1973) Angiographic changes in aortocoronary vein grafts: Lack of progression beyond the first year. Circulation, 48, 633
Levine, J.A., Bechtal, D.J., Gorlin, R., Cohn, P.F., Herman, M.P., CohN, L.H. \& Collins, J.J., Jr (1975) Coronary artery anatomy before and after direct revascularization surgery; clinical and ciné-arteriographic studies in 67 selected patients. American Heart Journal, 89, 561.

Malinow, M.R., Kremkau, E.L., Kloster, F.E., Bonchek, L.I. \& Rosch, J. (1973) Occlusion of coronary arteries after vein bypass. Circulation, 47, 1211.

Moran, S.V., Tarazi, R.C., Urzua, J.U., Favoloro, R.G. \& EFFLER, D.B. (1973) Effects of aortocoronary bypass on myocardial contractility. Journal of Thoracic and Cardiovascular Surgery, 65, 335.

Oldham, H.N., Jr, Roe, C.R., Young, W.G., Jr \& Dixon, S.H., Jr (1973) Intraoperative detection of myocardial damage during coronary artery surgery by plasma creatinine phosphokinase isoenzyme analysis. Surgery, 74, 917.

Sharma, B., Raphael, M.R., Goodwin, J.F., Rainbow, G. \& TAYLOR, S.H. (1976) Left ventricular angiography on exercise; a new method of assessing left ventricular function in patients with ischaemic heart disease. British Heart Journal (in press).

Sheldon, W.C., Rincon, G., Effler, D.B., Proudfit, W.L. \& MASON SONES, F., Jr (1973) Vein graft surgery for coronary artery disease. Survival and angiographic results in 1000 patients. Circulation, 48 (Suppl. 3), 184.

ShePherd, R.L., Itsioitz, S.B., Glancy, D.L., Stinson, E.B., Rees, R.L., Olinger, G.N., Clark, C.E. \& Epstein, S.E. (1974) Deterioration of myocardial function following aorto-coronary bypass operation. Circulation, 49, 467.

Solignac, A., Guerat, P. \& Bourassa, M.G. (1975) Influence of left ventricular function on survival three to four years after aortocoronary bypass. European Journal of Cardiology, 2, 421.

Wilhelmsson, C., Vedin, J.A., Wilhelmsen, L., Tibblin, G. \& WeRKo, L. (1974) Reduction of sudden death after myocardial infarction by treatment with alprenolol. Lancet, ii, 1157. 